\title{
Advances in Composites Based on the Palygorskite Mineral Nanofibers
}

\author{
Zhuo LIU ${ }^{1, a^{*}}$, Xu-Dong WANG ${ }^{1, b}$ and Jin WU ${ }^{2, c}$ \\ ${ }^{1}$ Automobile and Aviation College, Tianjin Sino-Geman University of Applied Sciences, Tianjin 300350, \\ China \\ ${ }^{2}$ Mechanical and Material College, Tianjin Sino-Geman University of Applied Sciences, Tianjin 300350, \\ China

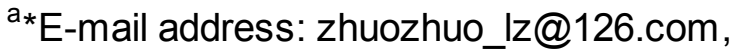 \\ ${ }^{b}$ E-mail address: xudong411@126.com, \\ 'E-mail address: wujin_0810@163.com.
}

Keywords: Palygorskite; Mineral nanofibers; Composites; Research progress

\begin{abstract}
Synthetic nanomaterials have the disadvantages of large-scale investment, high energy consumption, complex production process and heavy environmental load. Palygorskite mineral nanofibers are typical porous materials widely existing in nature and have the advantage of high surface area, moderate cation exchange capacity and excellent salt resistance, which have been widely applied as adsorbents in the dyes' removal from waste water. Due to the aggregation of palygorskite crystal bundles, it is difficult to totally release the adsorption capacity of palygorskite. Therefore, it is necessary to modify the microscopic structure of palygorskite with various physical or chemical techniques. Based on the above reason, this paper briefly summarizes the palygorskite-based composites research progress of palygorskite mineral nanofibers, and related research orientations have also been proposed.
\end{abstract}

\section{Introduction}

Palygorskite is a naturally available layer- ribbon porous hydrated magnesium aluminum silicate clay mineral, belonging to orthorhombic monoclinic system with the theoretical formula of $\mathrm{Mg}_{2} \mathrm{Al}_{2} \mathrm{Si}_{8} \mathrm{O}_{20}(\mathrm{OH})_{2}$ $\left(\mathrm{OH}_{2}\right)_{4} \cdot 4 \mathrm{H}_{2} \mathrm{O}[1,2]$. The layer-ribbon porous structure is formed via alternately stacking $\mathrm{Mg}, \mathrm{Al}, \mathrm{O}$ octahedral layer and $\mathrm{Si}, \mathrm{O}$ tetrahedral layer, making it many physical and chemical properties due to the special structure features of palygorskite [3-6].

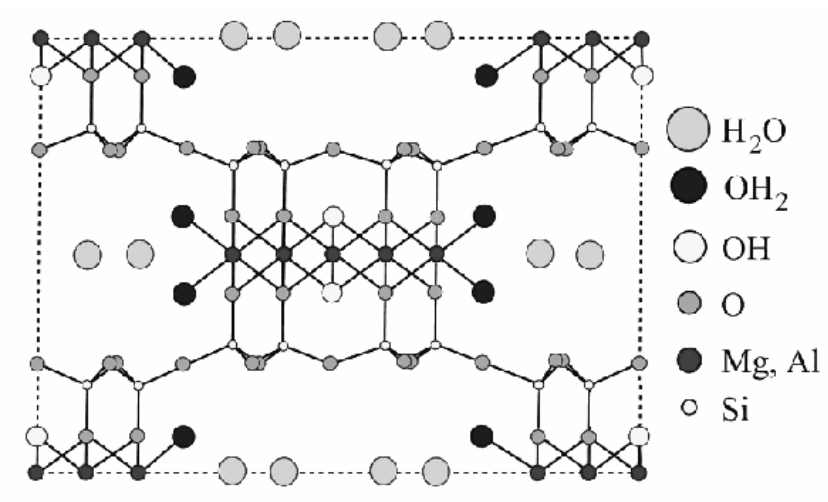

Fig.1 Diagram of crystal structure of palygorskite 
The diagram of crystal structure of palygorskite is shown in Fig. 1. Basic construction unit of palygorskite is composed of double ribbons of SiO4 tetrahedron, parallel to the c-axis. Each ribbon is connected to the next by the inversion of $\mathrm{SiO} 4$ tetrahedron along the oxygen atom. This kind of arrangement makes the tetrahedrons continuously bonded via the ribbons, thereby composing of ribbons of phyllosilicate [7-9]. From the cross section vertical to the c-axis, the octahedron is sandwiched by two tetrahedron layer units to constitute the layer- ribbon units, forming zeolite-like channels with a size of about $0.38 \mathrm{~nm} \times 0.63 \mathrm{~nm}$ via the alternate arrangement of layer-ribbon units and channel units $[10,11]$. Palygorskite has the peculiar structure, which contains zeolite water channels and pores making organic molecules and ions entering the inner structure of palygorskite, and exhibits good adsorption properties, rheological properties and catalytic performance $[12,13]$.

\section{Palygors kite-Based Composites for the Adsorption of Dyes}

In practice, some simple operation processes are applied in the preliminary process of the raw mineral to improve the adsorption and slurrying properties. However, it is limited that the adsorption capacity of palygorskite toward dyes can not be enhanced via these traditional methods. It is indispensable to adopt other treatments to modify the microstructure of palygorskite for the improvement of adsorption capacity, such as acid-activation, alkali-activation, thermal treatment, hydrothermal treatment and solvothermal treatment [14].

In recent decades, many reports mainly focus on the preparation pf palygorskite-based composites in order to improve the adsorption capacity and the active adsorption sites of palygorskite.

\section{Clay/Palygors kite Composites}

It is scarcely reported that the clay composite adsorbents consist of palygorskite and other clay minerals for the adsorption of dyes. A kind of mineral aggregate in natural sediments named diatomite is usually composed of amorphous silica and some other minerals. Due to the high porosity, diatomite displays excellent absorbability [15]. Based on the characteristics of diatomite and palygorskite, the palygorskite/diatomite composites are prepared by compounding, granulation, calcination and activation [16].

Bentonite is one kind of natural two-dimensional phyllosilicates with high specific surface area and high cation exchange property [17]. Meanwhile, the type of salt is adopted to fix dyes on the textile, but the salt resistance and hydration rate of palygorskite is higher than that of bentonite.

\section{Carbon/Palygorskite Composites}

Activated carbon has been worldwide introduced as adsorbents for the organic or inorganic pollutants' removal from the waste water treatment through the shift from the liquid phase to the solid phase [18]. However, the application of activated carbon as adsorbents in the waste water treatment is limited due to the high fabrication costs, which is motive to explore low-cost precursors for the replacement of the costly activated carbon $[19,20]$.

As a novel adsorbent, carbon/clay mineral composite has paid substantial attention to the potential applications of the water treatment [21]. In recent decades, Wu's group [22] focused on the preparation and properties of one-dimensional carbon/palygorskite composites. In 2013, the modification of palygorskite and the study on morphology and adsorption properties were carried out via a series of biomass carbon sources incorporating xylose, fructose, sucrose and cellulose via hydrothermal method [23]. Liu et al. [24] addressed the fabrication of carbon/palygorskite nanocomposites through a facile one-pot hydrothermal process in the existence of palygorskite and D-xylose. Anadão et al. [25] prepared the carbon/montmorillonite composites, which were derived from the used bleaching montmorillonite salt for the adsorption of MB and gasolin. Wang's group [26, 27] prepared carbon/palygorskite composites by one-step carbonization process with the palygorskite -based spent bleaching earth as the raw materials. 


\section{Palygorskite-Based Composite Hydrogels for the Adsorption of Dyes}

Hydrogel is a physically or chemically cross-linked natural or synthetic 3D network, and the hydrophilic structure renders them capable of holding large amounts of water in their three-dimensional networks [28]. Since hydrogels have the unique properties and numerous hydrophilic groups (amido, amino, carboxyl, hydroxyl, etc.) in the polymer chains, this series of hydrogels have been employed in a variety of industries, such as agriculture (water-reserving in soil and controlled release of agrochemicals), drug delivery system, separation technology, sludge/coal dewatering and so on [29, 30]. Recently, the trend of research has engaged in the integration of functional nanoparticles, such as quantum dot, metal or metal oxide and carbon-based nanocomposites so as to obtain hybrid composite hydrogels with fluorescene, magnetism, dielectric and catalytic properties for a large amount of practical usages [31-33]. The conclusion has been drawn that the composites of hydrogels not only overwhelm the disadvantages of hydrogels by combining the advantages of constituents, but also reduce the capital of the industrial polymers.

The superiority of clay-based hybrid hydrogels is obvious for removal of heavy metal ions, dyes and other pollutants. First, the assembly of the palygorskite-based materials into hydrogels can cut down the cost of water treatment, which vastly impacts the improvement of water treatment industry. Second, the diversity of clay materials leads to different kinds of hybrid hydrogels with specific pore structure, which contributes to the adsorption capacity towards pollutants. Third, the incorporation of clay minerals can improve the mechanical properties of hydrogels, which makes the recycling of adsorbents into reality. The presented palygorskite-based hydrogels are comprised of bulk hydrogels, granular hydrogels and environmental-sensitive intelligent hydrogels.

\section{Bulk Hydrogels}

During the synthesis process of hydrogel, palygorskite can be integrated into the polymeric network to form a loose and porous surface. The hybrid hydrogels have the properties of various adsorption sites, high adsorption rate and low cost of the preparation process. Li et al. [34] firstly synthesized poly (acrylic acid) / palygorskite superabsorbent composite by graft copolymerization reaction of acrylic acid on palygorskite using $\mathrm{N}, \mathrm{N}$-methylenebisacrylamide and ammonium persulfate as crosslinker and initiator, respectively. In order to improve the water absorbency of a superabsorbent in saline solution, the nonionic monomer of good salt-resistant acrylamide was introduced into poly (acrylic acid)/ palygorskite composites to prepare the poly (acrylic acid-co-acrylamide)/ palygorskite composites. Wang et al. [35, 36] studied on the adsorption properties of the (acrylic acid-co-acrylamide)/ palygorskite composites towards methyl violet and prepare chitosan-g-poly (acrylic acid) / palygorskite composites for the removal of methylene blue from aqueous solution.

Based on the above research achievements, the fabrication of the palygrskite-based hybrid hydrogels incorporated the organic crosslinker. Therefore, Liu et al. [37,38] improved the approach to synthesize the palygorskite/poly (acrylic acid) composite microgels via the one-pot radical polymerization with multi-functionalized palygorskite as crosslinker, and the prepared composites exhibited better mechanical stabilities and selective adsorption to heavy metal irons, especially to $\mathrm{Pb}^{2+}$.

\section{Granular Hydrogels}

The usual polymerization techniques such as emulsion polymerization, suspension polymerization, dispersion polymerization and precipitation polymerization have been adopted to synthesize the granular hydrogels [39]. However, during the reaction process, a large amount of toxic organic solvents, surfactants and dispersants are by-products, which may bring about the rise of the production cost, the environmental pollution, etc.

In recent decades, Wang's group [40] dedicated in the fabrication of granular hydrogels and clay-based hybrid hydrogels and the vastly usage of adsorption heavy metal ions, rare earth ions and dyes. Additionally, the oxidation-reduction initiator system composed of vitamin $\mathrm{C}$ and $\mathrm{H}_{2} \mathrm{O}_{2}$ was also successfully chosen to fabricate the granular hydrogels, which could get rid of the influences of iron element on the particles [41]. 


\section{Summary and Proposals in Future}

Based on the studies on comprehensive literatures, the conclusion can be made as follows. Firstly, the original separation methods of adsorbents from solution are filtration, sedimentation and centrifugation, and all of these methods are time-consuming and uneconomic. The magnetic separation technology has been demonstrated as a kind of effective, rapid and cheap separation technique to separate the palygorskite-based adsorbents from the solution after adsorption pollutants. Therefore, it is indispensable to explore the facile approach to prepare the palygorskite-based composites with functional groups to accomplish the high-efficiency adsorption and separation process. Secondly, the sustainable application of the spent palygorskite-based adsorbents loading of dye molecules or other pollutants should be taken into account. The last but not least, it is the inevitable trend in future to optimize the functionalization of adsorbents. In summary, although it has been explored that a variety of adsorbents are devoted to remove pollutants from aqueous solution, more studies need to be accomplished.

\section{Acknowledgements}

This research was financially supported by the Application Foundation and Advanced Technology Research Program of Tianjin city (Grant no. 14JCQNJC04100).

\section{References}

[1] M. Suarez, E. Garcia-Romero, FT-IR spectroscopic study of palygorskite: influence of the composition of the octahedral sheet, Appl. Clay Sci. 31 (2016), 154-163.

[2] Q.G. Tang, F. Wang, H. Guo, Y. Yang, Y.L. Du, J.S. Liang, F.Q. Zhang, Effect of coupling agent on surface free energy of organic modified attapulgite (OAT) powders and tensile strength of OAT/ethylene-propylene-diene monomer rubber nanocomposites, Powder Technol. 270 (2015), 92-97.

[3] M. Suárez, E. García-Romero, M.S. del Río, P. Martinetto, E. Dooryhée, The effect of octahedral cations on the dimensions of the palygorskite cell, Clay Miner. 42 (2007) 287-297.

[4] W.B. Wang, A.Q. Wang, Recent progress in dispersion of palygorskite crystal bundles for nanocomposites, Appl. Clay Sci. 119 (2016) 18-30.

[5] F. Wang, J. S. Liang, Q. G. Tang, et al, Preparation and far infrared emission properties of natural sepiolite nanofibers, J. Nanosci. Nanotechnol. 10(2010) 2017-2022.

[6] L. Bouna, B. Rhouta, M. Amjoud, et al, Synthesis, characterization and photocatalytic activity of $\mathrm{TiO}_{2}$ supported natural palygorskite microfibers, Appl. Clay Sci. 52 (2011) 301-311.

[7] Q. G. Tang, Y. Yang, F. Wang, et al, Effect of acid on surface properties of modified attapulgite and performance of styrene butadiene rubber filled by modified attapulgite, Nanosci Nanotechnol Lett. 6 (2014) 231-237.

[8] F. Wang, L. Feng, Q. G. Tang, et al, Preparation and performance of cis-polybutadiene rubber composite materials reinforced by organic modified palygorskite nanomaterials, J Nanomater. 936838 (2013) $1-5$.

[9] W.C.Yan, D. Liu, D.Y. Tan, et al, FTIR spectroscopy study of the structure changes of palygorskite under heating, Spectrochimica Acta Part A: Molecular and Biomolecular Spectroscopy, 97 (2012) 1052-1057.

[10] D. Gantenbein, J. Schoelkopf, G.P. Matthews, et al, Determining the size distribution-defined aspect ratio of rod-like particles, Appl. Clay Sci. 53 (2011) 538-543. 
[11]F. Wang, J. S. Liang, Q. G. Tang, et al, Channel microstructure and thermal insulation mechanism of sepiolite mineral nanofibers, J. Nanosci. Nanotechnol. 14 (2014) 3937-3942.

[12] Liu P, Polymer modified clay minerals: a review. Appl. Clay Sci. 38 (2007) 64-76.

[13] J.P. Rath, T.K. Chaki, D. Khastgir, Development of Natural Rubber-Fibrous Nano clay attapulgite composites: the effect of chemical treatment of filler on mechanical and dynamic mechanical properties of composites, Procedia Chem. 4 (2012) 131-137.

[14] B. Mu, A.Q. Wang, Adsorption of dyes onto palygorskite and its composites: A review, J. Environ. Chem. Eng. 4 (2016) 1274-1294.

[15] P. Yuan, D.Q. Wu, H.P. He, Z.Y. Lin, The hydroxyl species and acid sites on diatomite surface: a combined IR and Raman study, Appl. Clay Sci. 227 (2004) 30-39.

[16] Z. Wang, L.S. Zhang, Z.Q. Jing, Removal of Brilliant green from aqueous solution using diatomite-attapulgite composite nano-size adsorbent, Key Eng. Mater. 419-420 (2010) 525-528.

[17] J. Konta, Clay and man: Clay raw materials in the service of man, Appl. Clay Sci. 10 (1995) 275-335.

[18] N. Kannan, M.M. Sundaram, Kinetics and mechanism of removal of methylene blue by adsorption on various carbons- a comparative study, Dyes Pigm. 51 (2001)25-40.

[19] M. Rafatullah, O. Sulaiman, R. Hashim, et al, Adsorption of methylene blue on low-cost adsorbents: a review, J. Hazard. Mater, 177 (2010) 70-80.

[20] M.A. Khan, B.H. Hameed, J. Lawler, et al, Developments in activated functionalized carbons and their applications in water decontamination: a review, Desalin. Water Treat. 54 (2015) 422-449.

[21] R. Leboda, B. Charmas, J. Skubiszewska-Zięba, et al, Carbon-mineral adsorbents prepared by pyrolysis of waste materials in the presence of tetrachloromethane, J. Colloid Interface Sci. 284 (2005) 39-47.

[22] X.P. Xu, W.Y. Zhu, X.L. Zhang, et al, Catalytic deposition of nanocarbon onto palygorskite and its adsorption of phenol, Appl. Clay Sci. 52 (2011) 400-406.

[23] Y.Q. Xu, X.P. Wu, C. Liu, et al, Organic modification of palygorskite with biomass by hydrothermal carbonization and characterization on its adsorption property, Chem. React. Eng. Technol. 29 (2013) 119-124.

[24] W.J. Liu, T. Yang, J. Xu, et al, Preparation and adsorption property of attapulgite/carbon nanocomposite, Environ. Prog. Sustain Energy 34 (2015) 437-444.

[25] P. Anadão, I.L.R. Pajolli, E.A. Hildebrando, et al, Preparation and characterization of carbon/montmorillonite composites and nanocomposites from waste bleaching sodium montmorillonite clay, Adv. Powder Technol. 25 (2014) 926-932.

[26] J. Tang, B. Mu, M.S. Zheng, et al, One-step calcination of the spent bleaching earth for the efficient removal of heavy metal ions, ACS Sustain. Chem. Eng. 3 (2015) 1125-1135.

[27] J. Tang, B. Mu, L. Zong, et al, Fabrication of attapulgite/carbon composites from the spent bleaching earth for the efficient adsorption of methylene blue, RSC Adv. 5 (2015) 38443-38451.

[28] W.E. Hennink, C.F. van Nostrum, Novel crosslinking methods to design hydrogels, Adv. Drug Deliver. Rev. 64 (2012) 223-236.

[29] K. Habiri, H. Omidian, M.J. Zohuriaan-Mehr, et al, Superabsorbent hydrogel composites and nanocomposites: a review, Polym. Compos. 32 (2011) 277-289. 
[30] E.M. Ahmed, Hydrogel Preparation, characterization, and application: a review, J. Adv. Res. 6 (2015) $105-121$.

[31] S. Merino, C. Martín, K. Kostarelos, et al, Nanocomposite hydrogels: 3D polymernanoparticle synergies for on-demand drug delivery, ACS Nano 9 (2015) 4686-4697.

[32] Y.X. Xu, Z.Y. Lin, X.Q. Huang, et al, Functionalized grapheme hydrogel-based high-performance supercapacitors, Adv. Mater. 25 (2013) 5779-5784.

[33] Y.A. Zheng, A.Q. Wang, Ag nanoparticles-entrapped hydrogel as promising material for catalytic reduction of organic dyes, J. Mater. Chem. 22 (2012) 16552-16559.

[34] A. Li, A. Q. Wang, J.M. Chen, Studies on poly(acrylic acid)/ attapulgite superabsorbent composite, I. Synthesis and characterization, J. Appl. Polym. Sci. 92 (2004) 1596-1603.

[35] Y.S. Wang, L. Zeng, X.F. Ren, et al, Removal of Methyl Violet from aqueous solutions using poly(acrylic acid-co-acrylamide)/attapulgite composite, J. Environ. Sci. 22 (2010) 7-14.

[36] L. Wang, J.P. Zhang, A.Q. Wang, Fast removal of methylene blue from aqueous solution by adsorption onto chitosan-g-poly(acrylic acid)/attapulgite composite, Desalination 266 (2011) 33-39.

[37] P. Liu, L.P. Jiang, L.X. Zhu, et al, Attapulgite/poly(acrylic acid) nanocomposite (ATP/PAA) hydrogels with multifunctionalized attapulgite (org-ATP) nanorods as unique cross-liner: preparation optimization and selective adsorption of $\mathrm{Pb}(\mathrm{II})$ ion, ACS Sustain. Chem. Eng. 2 (2014) 643-651.

[38] P. Liu, L.P. Jiang, L.X. Zhu, et al, Novel approach for attapulgite /poly(acrylic acid ) (ATP/PAA) nanocomposite microgels as selective adsorbent for $\mathrm{Pb}$ (II) ion, React. Funct. Polym. 74 (2014) 72-80.

[39] Y.A. Zheng, A.Q. Wang, Superadsorbent with three-dimentional networks: from bulk hydrogel to granular hydrogel, Eur. Polym. J. 72 (2015) 651-686.

[40] Y.A. Zheng, A.Q. Wang, Granular hydrogel initiated by Fenton reagent and their performance on $\mathrm{Cu}(\mathrm{II})$ and Ni(II) removal, Chem. Eng. J. 200-202 (2012) 601-610.

[41] Y.A. Zheng, Y.F. Zhu, A.Q. Wang, Highly efficient and selective adsorption of malachite green onto granular composite hydrogel. Chem. Eng. J. 257 (2014) 66-73. 